\title{
New quantum Monte Carlo study of quantum critical phenomena with Trotter-number-dependent finite-size scaling and non-equilibrium relaxation
}

\author{
Yoshihiko Nonomura \\ Magnetic materials laboratory, Institute of physical and \\ chemical research (RIKEN), Wako, Saitama 351-0108, Japan*
}

PACS numbers: 05.70.Jk, 64.60.Ht, 75.10.Jm

\begin{abstract}
We propose a new efficient scheme for the quantum Monte Carlo study of quantum critical phenomena in quantum spin systems. Rieger and Young's Trotter-number-dependent finite-size scaling in quantum spin systems and Ito et al.'s evaluation of the transition point with the non-equilibrium relaxation in classical spin systems are combined and generalized. That is, only one Trotter number and one inverse temperature proportional to system sizes are taken for each system size, and the transition point of the transformed classical spin model is estimated as the point at which the order parameter shows power-law decay. The present scheme is confirmed by the determination of the critical phenomenon of the one-dimensional $S=1 / 2$ asymmetric XY model in the ground state. The estimates of the transition point and the critical exponents $\beta, \gamma$ and $\nu$ are in good agreement with the exact solutions. The dynamical critical exponent is also estimated as $\Delta=2.14 \pm 0.06$, which is consistent with that of the two-dimensional Ising model.
\end{abstract}

\section{Introduction}

Quantum critical phenomena are one of the most interesting topics in low-dimensional quantum spin systems, and various numerical methods have been proposed. In onedimensional systems, the exact-diagonalization method gives the most reliable result

* Present address: Computational materials science division, National research institute for metals (NRIM), Tsukuba, Ibaraki 305-0047, Japan 
when the chain is single and the size of spins is not so large. In multi-leg ladder systems or large-spin systems, reachable system sizes with this method are not satisfactory and other approximate methods should be used. Historically, the quantum Monte Carlo (QMC) method [1, 2] and related methods (the quantum transfer-matrix $\operatorname{method}[2,3$, 44, the Monte Carlo power method [2, 5] etc.) have been used, and nowadays the density-matrix renormalization-group (DMRG) method [6, 6, \&] is considered to be most powerful because quite large (effectively infinite) systems can be calculated with small errors and relatively small required CPU demand. The DMRG method is applicable even to two-dimensional systems with large enough energy gap, but the limitation of computer memories makes the estimation of critical phenomena difficult at present (any systems are gapless at the critical point in the thermodynamic limit). Then, the QMC method is still most useful to study quantum critical phenomena in non-frustrated two-dimensional quantum spin systems.

In QMC simulations of ground states in quantum spin systems, the most serious problem is large sampling errors. The QMC method is essentially applicable only to finite-temperature systems, and "ground states" mean nothing but "calculations at low enough temperatures", which might include ambiguity. Moreover, simulations at low temperatures result in large correlation time, which is the origin of large sampling errors. Furthermore, in the conventional world-line QMC method, physical quantities are evaluated after the extrapolation of the data for various Trotter numbers, which makes statistical errors larger. These shortcomings of the world-line QMC algorithm have been overcome with the stochastic series expansion method 9, 10, 11], the quantum cluster algorithm 12, 13] or Prokof'ev's algorithm[14, 15] proposed recently. The Trotter extrapolation is not required in these methods, and fast relaxation of them makes simulations at very low temperatures possible. As long as finite systems are considered, energy gaps are finite even at the transition point, and simulations at the temperatures lower enough than energy gaps give "ground-state" properties with convergent correlation time. Although such a brute-force strategy is required for the evaluation of actual physical quantities at the ground state, more efficient scheme is expected to study quantum critical phenomena, in which universal behavior exists.

In the present paper, we propose a new efficient scheme to study quantum critical phenomena in quantum spin systems with the QMC method. This scheme can be regarded as a combination and a generalization of two independent techniques of Monte Carlo calculations originally proposed in Ising spin systems. That is, Rieger and Young's Trotter-number-dependent finite-size scaling[16] introduced in the QMC study of the quantum critical phenomenon of the Ising spin glass model in a transverse field is coupled with Ito et. al.'s determination of the transition point [17] based on the non-equilibrium relaxation of the order parameter. The latter method was introduced in the classical Ising spin glass model, and the former method is necessary for the generalization to quantum spin systems. By use of the present scheme, only one Trotter number has to be considered in each system size for the determination 
of the universality class. In short, the combination of these two methods is essential for efficient numerical calculations in quantum spin systems.

In order to verify the applicability of the present scheme, we estimate the transition point and critical exponents of the one-dimensional $S=1 / 2$ asymmetric XY model. This model is mapped on a two-dimensional Ising spin model with conservation laws (or infinite multi-body interactions in the Ising-spin representation) by the Suzuki-Trotter decomposition [1], and the universality class of this model 18, 19, 20] is different from that of the two-dimensional Ising model. In section 2, the present scheme is explained together with a brief review of the world-line QMC method. In section 3 , the quantum critical phenomenon of the one-dimensional $S=1 / 2$ asymmetric XY model is analyzed. The transition point and the dynamical critical exponent are estimated with very large clusters (linear sizes are $L=1024,2048$ and 4096) and various critical exponents are estimated with the clusters up to $L=128$. The above descriptions are summarized in section 4. Part of the present results have already been briefly reported elsewhere 21].

\section{Numerical methods}

In the world-line quantum Monte Carlo method [1, 2], the partition function of quantum spin systems is transformed to a classical one by the Suzuki-Trotter formula,

$$
Z \equiv \operatorname{Tr} \mathrm{e}^{-\beta \mathcal{H}}=\lim _{M \rightarrow \infty} \operatorname{Tr}\left[\exp \left(-\frac{\beta}{M} \mathcal{H}_{1}\right) \exp \left(-\frac{\beta}{M} \mathcal{H}_{2}\right)\right]^{M}
$$

where $\beta$ stands for the inverse temperature $(\beta \equiv 1 / T)$, and the partial Hamiltonians $\mathcal{H}_{1}$ and $\mathcal{H}_{2}$ in one-dimensional systems are shown in figure 4 , which is known to be the checkerboard decomposition. Then, each 4-spin unit cell is independent, and Monte Carlo simulations are possible. Conventional local flips and the $x$-direction global flip are introduced. When the models have conservation laws, the Trotterdirection global flip should be considered for the evaluation of the thermal averages in the whole phase space. As is well known, the tangent-direction global flip can be safely omitted, and this algorithm is vectorized 22].

In the conventional world-line QMC method, quantities in the ground state are evaluated as follows:

1. The temperature is fixed at a "low enough" one, $T_{0}(L)$, which is much smaller than the energy gap of finite clusters, $\Delta E(L)$, in the subspace in which the ground state is included. The Trotter-direction global flip is not introduced in this case.

2. QMC simulations are performed for some finite Trotter numbers $M$, and quantities in the $M \rightarrow \infty$ limit are estimated with the following scaling form, $Q(M, L)=Q(\infty, L)+$ const. $/ M^{2}+\cdots$. 
3. Quantities in the thermodynamic limit are evaluated from the finite-size scaling of $\{Q(\infty, L)\}$ for various system sizes.

In the above analysis, the $T \rightarrow 0$ limit is skipped by use of the finite energy gap in finite systems, but double extrapolations with respect to $M$ and $L$ are still necessary. Calculations for some Trotter numbers are required for each system size, and simulations at a "low enough" temperature consume much CPU demand because of long correlation time.

Although the above analysis is required for the evaluation of a certain physical quantity in the ground state, such a complicated procedure is not necessary for the investigation of quantum critical phenomena. The universality class of the original $D$-dimensional quantum system in the ground state is expected to be equivalent to that of the transformed $(D+1)$-dimensional classical system, and Rieger and Young[16] pointed out that the finite-size scaling holds when the ratios $M / L^{z}$ and $\beta / M$ are fixed. The exponent $z$ stands for the ratio of the correlation length in the real direction $(\xi)$ and that in the Trotter direction $\left(\xi_{\tau}\right)$, namely $\xi^{z} \propto \xi_{\tau}$ in the vicinity of the transition point. This exponent is given by $z=1$ in non-random systems (the Lorentz invariance). In this scheme, the temperature is scaled in accordance with the system size, and the ambiguity of the simulated temperature is automatically removed. Moreover, this temperature can be higher than the standard "low enough" temperature.

The transition point has been estimated from physical quantities in equilibrium until present. When it is determined by the finite-size scaling analysis, the critical exponent should also be evaluated simultaneously, and the accuracy of estimates becomes poor. When it is estimated from scale-invariant quantities, the above disadvantage is excluded. However, statistical errors of such complicated quantities are larger than those of ordinary quantities, and the final estimates are not expected to be so accurate again. Then, in the present paper, we utilize a new method for the determination of the transition point recently proposed by Ito et. al. [17]. They showed that the transition point can be estimated quite accurately with the nonequilibrium relaxation of the order parameter. That is, simulations are started from a perfectly-ordered state, and the order parameter is measured in each Monte Carlo step (MCS). The order parameter decays exponentially in the disordered phase, remains non-vanishing in the ordered phase, and power-law decay is observed only at the transition point (figure 4 ). This calculation uses only several hundreds or a few thousands of MCS in each sample, and very large systems can be easily treated without equilibration and Monte Carlo sampling.

Since the Trotter extrapolation is justified only in equilibrium quantities, this method is not consistent with the conventional world-line QMC method. Thus, the combination with Rieger and Young's scheme is indispensable for applications to quantum spin systems. Since the transition point is not a universal value, the 
estimate obtained from transformed classical spin models with fixed $\beta / M$ is generally different from the transition point of the original quantum spin model. However, the transition point of the transformed model should be used in the finite-size-scaling analysis of a series of systems with fixed $\beta / M$, and the "true" transition point of the original model is estimated from some simulations with different values of $\beta / M$. Details of this analysis will be explained elsewhere 23.

The present scheme is so general that it can be coupled with any kinds of Monte Carlo dynamics in principle. Although values of the dynamical critical exponent depend on the dynamics (details will be explained in the next section), the powerlaw-decay behavior at the transition point is common as long as second-order phase transitions are considered. The essential point of the usage of Rieger and Young's scheme in the present framework is the idea that simulated temperatures are scaled in accordance with variance of system sizes. Thus, Trotter-number-free methods such as the continuous-time loop algorithm 13 can be utilized. On that occasion, $\beta / L^{z}$ should be fixed instead of $M / L^{z}$ and $\beta / M$. On the other hand, the accuracy of the estimate of the transition point depends on the dynamics. If a very fast dynamics such as the loop algorithm is utilized, the decay process is completed within first several MCS and precise evaluation of the transition point would be difficult because of large fluctuations of the order parameter and the discontinuity of MCS. Of course, this statement is also model dependent: even the loop algorithm might be useful in systems with principal slow dynamics such as quantum spin glass models. We can at least tell that the conventional "slow" dynamics is suitable for regular systems as will be shown in the next section.

\section{Numerical calculations of critical phenomenon}

In order to verify the applicability of the new scheme explained in the previous section, we analyze the quantum critical phenomenon of the one-dimensional $S=1 / 2$ asymmetric XY model described by the following Hamiltonian,

$$
\mathcal{H}=-\sum_{i=1}^{L} S_{i}^{x} S_{i+1}^{x}-g \sum_{i=1}^{L} S_{i}^{y} S_{i+1}^{y}-H^{x} \sum_{i=1}^{L} S_{i}^{x},
$$

with the periodic boundary condition $\left(S_{L+1}^{x, y}=S_{1}^{x, y}\right)$. The in-plane field $H^{x}$ is introduced only for the definition of the zero-field susceptibility, and it is not applied in actual simulations. We only consider even-spin systems which have a singlet ground state. This is the simplest quantum spin model with conservation laws, and this model has already been solved exactly 18, 19, 20]. There are no matrix elements between the two subspaces with $\sum_{i} S_{i}^{x}=$ odd and $\sum_{i} S_{i}^{x}=$ even. The apparent transition point $g_{\mathrm{c}}=1$ is common in the original model (2) and the transformed classical spin models. Simulations are based on the up-down basis along the $x$-direction, 
and the Trotter number and simulated temperature are fixed as $2 M / L=1$ and $\beta / M=1$, following Rieger and Young's parameterization 16. Under these conditions, the original $L$-spin system is transformed to a classical $L \times L$-spin system with all the interactions unity at $g=1$, and good scaling behavior is expected. The phase space is limited to the one with $\sum_{i} S_{i}^{x}=$ even in which the ground state is included. Since finite (not "low enough") temperatures are introduced in the present scheme, the above restriction of the phase space cannot be justified a priori. Complementary simulations without this restriction are discussed in Appendix.

\subsection{Transition point $g_{\mathrm{c}}$}

First, the transition point is estimated with the non-equilibrium relaxation of the order parameter. When the $x$-component of the magnetization,

$$
m^{x}(L) \equiv \frac{1}{L} \sum_{i=1}^{L} S_{i}^{x},
$$

is regarded as the order parameter, the regions $g<1$ and $g>1$ correspond to the ordered and disordered phases, respectively. Simulations are started from the all-up state, and the initial $3 \times 10^{3}$ Monte Carlo steps (MCS) are measured for the $L=1024,2048$ and 4096 systems. In simulations for these system sizes, data with 20, 5 and 1 independent sequences of random numbers are averaged, respectively. The order parameter of the $L=4096$ system is plotted versus MCS in a logarithmic scale in figure $4(\mathrm{a})$, and this behavior is the same as displayed in figure 4 . That is, the data at $g=1.000$ are almost on a straight line, and the data at $g=0.996$ and $g=1.004$ are clearly upward- and downward-bending, respectively. Therefore, the transition point is estimated as $g_{\mathrm{c}}=1.000 \pm 0.002$ within the present simulations, as expected. The data for the $L=1024$ and 2048 systems at $g=1.000$ are given in figure 1(b) together with those for the $L=4096$ system, and this figure means that there exists no explicit systematic size dependence in such large systems. Since values of $L \times 2 M$ spins are averaged in these simulations, the data show relatively smooth behavior in spite of small number of averaged random-number sequences.

Next, we perform similar simulations in smaller systems. In equilibrium calculations, the "approximate" transition point $g_{\text {ap }}(L)$ such as the maximum of the specific heat shows the following systematic deviation from the transition point $g_{\mathrm{c}}$ in finite systems,

$$
g_{\text {ap }}(L)=g_{\mathrm{c}}+\text { const. } \times L^{1 / \nu}+\cdots,
$$

and the critical exponent of the correlation length, $\nu$, is estimated. The relaxation of the order parameter for $L=32$ and 64 (the numbers of averaged samples are 16384 and 4096, respectively) is displayed in figures $4(\mathrm{a})$ and $1(\mathrm{~b})$, respectively. These figures show that the order parameter decays exponentially after a certain MCS even for $g<1$. This property is quite natural because the order parameter vanishes 
regardless of the value of $g$ in finite systems in equilibrium. These figures also reveal that the crossover MCS from "quasi-infinite" properties to finite-size ones depends on system sizes, namely $\sim 40$ MCS for $L=32$, and $\sim 180$ MCS for $L=64$. These results suggest that the above behavior of $L>1000$ systems is nothing but transient, but that the present $3 \times 10^{3}$ MCS are small enough to be regarded as "quasi-infinite" time scale. Moreover, the transition point estimated from these figures is also $g_{\mathrm{c}} \simeq 1.00$, and the size dependence of the estimates seems quite small. This behavior is not unphysical because the "quasi-infinite" property means that finite-size effects are not apparent in this time scale. Thus, the scaling form (4) is not useful for the estimation of the critical exponent $\nu$ within the present scheme.

As explained in the previous section, the "transition point" estimated with the present scheme generally depends on the value of $\beta / M$. On the other hand, it is independent of the value of $M / L$, because "quasi-infinite" properties are observed in the present scheme and the difference of the shape of transformed clusters from squares is expected to result only in the difference the crossover MCS as shown above. Then, in order to confirm this argument, we simulate systems with (a) $4 M / L=1$ ( $L=4096, M=1024,2$ samples) and (b) $M / L=1$ ( $L=2048, M=2048,2$ samples). These results are plotted versus MCS in a logarithmic scale in figures 1 (a) and $4(\mathrm{~b})$, respectively. These figures also give $g_{\mathrm{c}}=1.000 \pm 0.002$, as expected.

\subsection{Dynamical critical exponent $\Delta$}

According to the dynamical finite-size scaling theory 24, 25, the non-equilibrium relaxation of the order parameter at the transition point is scaled [26, 27] as

$$
m^{x}(t) \sim t^{-\lambda}, \quad \lambda=\frac{\beta}{\Delta \nu}
$$

where $t$ stands for MCS, the critical exponents $\beta$ and $\nu$ will be defined in the next subsection ((14) and (16)), and the dynamical critical exponent $\Delta$ is defined as

$$
\begin{aligned}
& \left\langle S_{i}^{x}(0) S_{i}^{x}(t)\right\rangle-\left\langle S_{i}^{x}(0)\right\rangle^{2} \sim \exp (-t / \tau), \\
& \tau \sim\left|g-g_{\mathrm{c}}\right|^{-\Delta} \text { for } g \rightarrow g_{\mathrm{c}} .
\end{aligned}
$$

Although the transition point can be estimated accurately enough with the data displayed in figure $4(\mathrm{a})$, these data are too divergent to evaluate the exponent $\lambda$ with the local-exponent method originally proposed by Stauffer 26. Then, we naively fit the data shown in figure 4 (a) with the scaling form (5) for various time scales $\left(t=t_{\text {ini }} \sim t_{\text {fin }}, \Delta t \equiv t_{\text {fin }}-t_{\text {ini }}=50,100\right.$ and 200). Estimates of the exponent $\lambda$ are plotted versus $1 / t_{\text {mid }}$ in figure $甘$, with $t_{\text {mid }} \equiv\left(t_{\text {ini }}+t_{\text {fin }}\right) / 2$. In addition to the data given in figure $4(\mathrm{a})$, results of two more independent simulations are averaged in order to obtain reliable estimates. In each $\Delta t, \lambda$ almost remains constant in the early stage, and it begins to fluctuate as $t_{\text {mid }}$ becomes larger. As for the final 
estimate $\lambda_{\text {est }}$, we require that the central value should be close to the early-stage one, and that initial up-down fluctuations should be included within the error bar. These conditions are satisfied when we take $\lambda_{\text {est }}=0.1167 \pm 0.0033$ (see figure 4 ). Combining this exponent with the exact solution, $\beta / \nu=0.25[18$, 19, 20], the dynamical critical exponent of the present model is estimated as

$$
\Delta=2.14 \pm 0.06
$$

which is consistent with that of the two-dimensional Ising model, $\Delta=2.165 \pm$ 0.010 28. This result might suggest that the dynamical critical exponent is common in the free-fermion models, even though the universality classes are different. Actually, the critical exponent $\nu$ defined by

$$
\begin{aligned}
& \left\langle S_{i}^{x} S_{j}^{x}\right\rangle-\left\langle S_{i}^{x}\right\rangle^{2} \sim \exp \left(-\left|r_{i}-r_{j}\right| / \xi\right), \\
& \xi \sim\left|g-g_{\mathrm{c}}\right|^{-\nu} \quad \text { for } \quad g \rightarrow g_{\mathrm{c}},
\end{aligned}
$$

is unity in both the present model and the two-dimensional Ising model. When the definition of $\Delta$, (6) and (7), is compared with that of $\nu$, (9) and (10), the exponent $\Delta$ can be regarded as the MCS version of $\nu$. Since these two models have the common $\nu$, it is not strange that they might also have the common $\Delta$. Of course, the statistical error of the present estimate is not small enough, and further studies in this direction (averaging much sequences of random numbers or simulating larger systems) are required.

\subsection{Critical exponents $\beta / \nu$ and $\gamma / \nu$}

Next, we estimate the critical exponents which can be obtained from equilibrium simulations at $g=g_{\mathrm{c}}=1$. We evaluate the squared magnetization defined by

$$
m^{2}(g, L) \equiv\left\langle\left(m^{x}(L)\right)^{2}\right\rangle=\frac{1}{2 M L^{2}} \sum_{n=1}^{2 M}\left\langle\left(\sum_{i=1}^{L} S_{i, n}^{x}\right)^{2}\right\rangle,
$$

and the zero-field susceptibility for the in-plane field defined by

$$
\left.\chi(g, L) \equiv \frac{\partial}{\partial H^{x}}\left\langle m^{x}(L)\right\rangle\right|_{H^{x}=0}=\frac{\beta}{4 M^{2} L}\left\langle\left(\sum_{i=1}^{L} \sum_{n=1}^{2 M} S_{i, n}^{x}\right)^{2}\right\rangle .
$$

Here $n$ stands for the suffix in the Trotter direction, and these quantities show the following scaling behavior at the transition point,

$$
m^{2}\left(g_{\mathrm{c}}, L\right) \sim L^{-2 \beta / \nu}, \quad \chi\left(g_{\mathrm{c}}, L\right) \sim L^{\gamma / \nu}
$$

where the critical exponents $\beta, \gamma$ and $\nu$ are defined as

$$
\begin{aligned}
m(g, \infty) & \sim\left(g_{\mathrm{c}}-g\right)^{\beta} \quad \text { for } g \rightarrow g_{\mathrm{c}}, \\
\chi(g, \infty) & \sim\left|g-g_{\mathrm{c}}\right|^{-\gamma} \text { for } g \rightarrow g_{\mathrm{c}}, \\
\xi(g, \infty) & \sim\left|g-g_{\mathrm{c}}\right|^{-\nu} \text { for } g \rightarrow g_{\mathrm{c}} .
\end{aligned}
$$


Table 1: Monte Carlo steps for equilibration (E-MCS) and for measurement (MMCS) are listed for various system sizes.

\begin{tabular}{rcc}
\hline$L$ & E-MCS & M-MCS \\
\hline 16 & $0.2 \times 10^{6}$ & $0.2 \times 10^{7}$ \\
24 & $0.4 \times 10^{6}$ & $0.4 \times 10^{7}$ \\
32 & $0.8 \times 10^{6}$ & $0.8 \times 10^{7}$ \\
48 & $1.2 \times 10^{6}$ & $1.2 \times 10^{7}$ \\
64 & $0.5 \times 10^{6}$ & $0.5 \times 10^{7}$ \\
80 & $0.8 \times 10^{6}$ & $0.8 \times 10^{7}$ \\
96 & $1.0 \times 10^{6}$ & $1.0 \times 10^{7}$ \\
112 & $1.2 \times 10^{6}$ & $1.2 \times 10^{7}$ \\
128 & $2.0 \times 10^{6}$ & $2.0 \times 10^{7}$ \\
\hline
\end{tabular}

Simulations are performed for the $L=16,24,32,48,64,80,96,112$ and 128 systems, and the MCS for each system size are listed in table 11. Results of these calculations are given in table 2 and figures 1 (a) and $1(\mathrm{~b})$. The critical exponents are estimated by the least-squares fitting of the data for $64 \leq L \leq 128$ with the scaling forms (13) as

$$
\beta / \nu=0.241 \pm 0.003, \gamma / \nu=1.499 \pm 0.005 .
$$

On the other hand, when we utilize the following scaling forms in which the nextorder correction terms are considered,

$$
\begin{aligned}
m^{2}\left(g_{\mathrm{c}}, L\right) & =a_{1} L^{-2 \beta / \nu}+a_{2} L^{-2 \beta / \nu-1}+\cdots, \\
\chi\left(g_{\mathrm{c}}, L\right) & =b_{1} L^{\gamma / \nu}+b_{2} L^{\gamma / \nu-1}+\cdots,
\end{aligned}
$$

we have

$$
\beta / \nu=0.245 \pm 0.006, \gamma / \nu=1.49 \pm 0.02,
$$

from the least-squares fitting of the data for $24 \leq L \leq 128$. The estimates (17) and (20) are both consistent with the exact values, $\beta / \nu=0.25$ and $\gamma / \nu=1.5[18$, 19, 20].

\subsection{Critical exponent $\nu$}

The critical exponents $\beta / \nu$ and $\gamma / \nu$ are related 29] to one another through the exponent $z$ as

$$
2 \beta / \nu+\gamma / \nu=d+z
$$

where $d$ denotes the spatial dimension. Then, the critical exponent $\nu$ should be estimated independently for the complete determination of the universality class. 
Table 2: Ground-state averages of the squared magnetization and the susceptibility at the transition point for various system sizes.

\begin{tabular}{rclc}
\hline$L$ & $m^{2}$ & $\chi$ \\
\hline 16 & $0.11094 \pm 0.00031$ & $1.2242 \times 10^{1} \pm 3.0 \times 10^{-2}$ \\
24 & $0.09379 \pm 0.00035$ & $2.2785 \times 10^{1} \pm 6.5 \times 10^{-2}$ \\
32 & $0.08279 \pm 0.00037$ & 3.531 & $\times 10^{1} \pm 1.2 \times 10^{-1}$ \\
48 & $0.06868 \pm 0.00023$ & 6.589 & $\times 10^{1} \pm 3.0 \times 10^{-1}$ \\
64 & $0.06052 \pm 0.00029$ & $1.0299 \times 10^{2} \pm 6.3 \times 10^{-1}$ \\
80 & $0.05437 \pm 0.00021$ & $1.4385 \times 10^{2} \pm 7.2 \times 10^{-1}$ \\
96 & $0.04982 \pm 0.00026$ & 1.890 & $\times 10^{2} \pm 1.3 \times 10^{0}$ \\
112 & $0.04624 \pm 0.00026$ & 2.381 & $\times 10^{2} \pm 1.7 \times 10^{0}$ \\
128 & $0.04336 \pm 0.00022$ & 2.912 & $\times 10^{2} \pm 1.9 \times 10^{0}$ \\
\hline
\end{tabular}

This calculation requires several simulations out of the transition point, and they consume much more CPU time than the simulations in the previous subsection. Since the direct evaluation of the correlation length is not so accurate, we instead estimate the critical exponent $\nu$ on the basis of the following scaling functions,

$$
\begin{aligned}
m^{2}(g, L) & \sim L^{-2 \beta / \nu} f(L / \xi(g)) \sim L^{-2 \beta / \nu} F\left(L^{1 / \nu}\left|g-g_{\mathrm{c}}\right|\right) \\
\chi(g, L) & \sim L^{\gamma / \nu} g(L / \xi(g)) \sim L^{\gamma / \nu} G\left(L^{1 / \nu}\left|g-g_{\mathrm{c}}\right|\right) .
\end{aligned}
$$

These formulas mean that the derivatives with respect to $g$ behave as

$$
\left.\frac{\mathrm{d}}{\mathrm{d} g} m^{2}(g, L)\right|_{g=g_{\mathrm{c}}} \sim L^{(1-2 \beta) / \nu},\left.\frac{\mathrm{d}}{\mathrm{d} g} \chi(g, L)\right|_{g=g_{\mathrm{c}}} \sim L^{(1+\gamma) / \nu} .
$$

Practically, such derivatives are replaced by the evaluation of the slopes of the physical quantities just below the transition point. For example, the susceptibility of the $L=128$ system is displayed in figure 4 . The exponents appeared in (24) are estimated as

$$
(1-2 \beta) / \nu=0.525 \pm 0.007, \quad(1+\gamma) / \nu=2.506 \pm 0.006
$$

by the least-squares fitting of the data for $80 \leq L \leq 128$ (see table 3 and figures 1 (a) and $4(\mathrm{~b})$ ) with (24). From the exponents (17) and (25), we have

$$
1 / \nu=1.007 \pm 0.008 \text { or } \nu=0.993 \pm 0.008
$$

assuming the statistical independence of the exponents (17) and (25). This estimate is consistent with the exact value, $\nu=1[20]$. Note that the complete coincidence of the estimates of $\nu$ obtained from two different quantities would be by chance. 
Table 3: Slopes of the squared magnetization and the susceptibility just below the transition point for various system sizes.

\begin{tabular}{rcc}
\hline$L$ & $m^{2}$ & $\chi$ \\
\hline 64 & $0.5942 \pm 0.0039$ & $1301 \pm 8$ \\
80 & $0.6804 \pm 0.0062$ & $2320 \pm 21$ \\
96 & $0.7590 \pm 0.0086$ & $3720 \pm 42$ \\
112 & $0.8191 \pm 0.0086$ & $5457 \pm 57$ \\
128 & $0.8714 \pm 0.0074$ & $7567 \pm 64$ \\
\hline
\end{tabular}

\section{Summary and future problems}

In the present paper, we have proposed a new efficient finite-size scaling scheme to study quantum critical phenomena in quantum spin systems on the basis of the quantum Monte Carlo method. Instead of the conventional Trotter extrapolation at low enough temperatures, we concentrate on transformed Ising spin systems with $2 M / L=1$ and $\beta / M=1$. The transition point of the transformed model is estimated with the non-equilibrium relaxation of the order parameter, and various critical exponents are evaluated from the standard finite-size scaling of transformed classical spin systems. That is, the critical exponents divided by the critical exponent of the correlation length, $\nu$, are estimated from the size dependence of physical quantities at the transition point. The exponent $\nu$ itself is evaluated from the size dependence of the slopes of physical quantities just below the transition point. Then, the universality class of the models are completely determined within the present framework. In order to verify the applicability of the present scheme, we have estimated the transition point and the critical exponents $\beta, \gamma$ and $\nu$ of the one-dimensional $S=1 / 2$ asymmetric XY model, and all the critical exponents have been evaluated with high accuracy in comparison with the exact solutions. The dynamical critical exponent has also been estimated as $\Delta=2.14 \pm 0.06$, which is consistent with that of the two-dimensional Ising model. The present estimation of the transition point is more efficient than any other methods based on equilibrium quantities. The present finitesize-scaling scheme is so general that it can be combined with improved algorithms such as the quantum cluster algorithm.

The present simulations have been performed in the restricted phase space with $\sum_{i} S_{i}^{x}=$ even in which the ground state is included. Complementary simulations without this restriction (simulations including the Trotter-direction global flip) have also been performed, and we have found that theses simulations require much $\mathrm{CPU}$ demand (about 1.3 times larger in the present model) and show larger finite-size 
corrections than those with the restriction of the phase space. These results mean that simulations including the Trotter-direction global flip are useless. We emphasize that the conventional "slow" single-spin-flip-type dynamics is practically useful for the precise estimation of the transition point in the present scheme. On the other hand, the introduction of the quantum cluster algorithm in simulations of equilibrium quantities is an important future task for us. Applications to quantum critical phenomena of the two-dimensional $S=1 / 2$ dimerized Heisenberg models 23 and the two-dimensional $S=1 / 2$ random Heisenberg models [30] will be reported in the near future.

\section{Acknowledgements}

The present author is supported by the Special Researcher's Basic Science Program from RIKEN. He would like to thank Professor S. Miyashita for valuable suggestions and useful comments. He used the vectorized random-number generation program RNDTIK developed by Professor N. Ito and Professor Y. Kanada. Most numerical calculations were performed on FACOM VPP500 at the Institute for Solid State Physics, University of Tokyo.

\section{Appendix}

Here we show the results of simulations including the Trotter-direction global flip. Since details of the analysis have already been explained in section 2 and 3, only the results for the transition point and the critical exponents $\beta / \nu$ and $\gamma / \nu$ are briefly summarized.

The transition point is estimated with the $L=4096$ system for one sequence of random numbers. The decay of the order parameter from the all-up state is measured during $3 \times 10^{3}$ MCS as shown in figure 4 , and we have $g_{\mathrm{c}}=1.000 \pm 0.002$. Although this estimate is the same as obtained in section 3, fluctuations of the order parameter become larger than those measured in section 3. This result means that the present scheme also works well when the Trotter-direction global flip is included, but the expansion of the phase space results in the increase of fluctuations, which is not desirable for accurate estimation of critical phenomena.

The squared magnetization and the zero-field susceptibility are simulated at the transition point $g=g_{\mathrm{c}}=1$ for the same system sizes and the same MCS as listed in table 1. The size dependence of these quantities are displayed in figures $4(\mathrm{a})$ and ๑(b), and the critical exponents are estimated with the scaling forms (13) as

$$
\beta / \nu=0.219 \pm 0.007, \gamma / \nu=1.546 \pm 0.009
$$


from the $64 \leq L \leq 128$ clusters, and with (18) and (19), we have

$$
\beta / \nu=0.22 \pm 0.03, \gamma / \nu=1.56 \pm 0.05
$$

from the $32 \leq L \leq 128$ clusters. These estimates are not inconsistent with the exact solutions, $\beta / \nu=0.25$ and $\gamma / \nu=1.5$, but the accuracy is not so good as the results obtained in section 3 due to larger finite-size corrections.

The above two numerical calculations with respect to non-equilibrium relaxation and equilibrium quantities are based on the same algorithm, and they consume larger CPU time than those in section 3 because of the extra Trotter-direction global flip. They spend about 1.3 times larger CPU demand in the present model. All the results suggest that simulations including the Trotter-direction global flip are inferior to those without that kind of global flip.

\section{References}

[1] Suzuki M 1976 Prog. Theor. Phys. 561454.

[2] Quantum Monte Carlo Methods in Condensed Matter Physics, ed M Suzuki (World Scientific, Singapore, 1993).

[3] Betsuyaku H 1984 Phys. Rev. Lett. 53629

[4] Betsuyaku H 1985 Prog. Theor. Phys. 73319

[5] Koma T 1993 J. Stat. Phys. 71269

[6] White S R 1992 Phys. Rev. Lett. 692863

[7] White S R 1993 Phys. Rev. B 4810345

[8] Nishino T and Okunishi K 1995 J. Phys. Soc. Japan 644084

[9] Sandvik A W and Kurkijärvi J 1991 Phys. Rev. B 435950

[10] Sandvik A W 1992 J. Phys. A 253667

[11] Sandvik A W 1997 Phys. Rev. B 5611678

[12] Evertz H G, Lana G and Marcu M 1993 Phys. Rev. Lett. 70875

[13] Beard B B and Wiese U-J 1996 Phys. Rev. Lett. 775130

[14] Prokof'ev N V, Svistunov B V and Tupitsyn I S 1996 Zh. Eksp. Teor. Fiz. 64 853, cond-mat/9703200

[15] Sandvik A W, Singh R R P and Campbell D K 1997 Phys. Rev. B 5614510 
[16] Rieger H and Young A P 1994 Phys. Rev. Lett. 724141

[17] Ito N, Matsuhisa T and Kitatani H 1998 J. Phys. Soc. Japan 671188

[18] Lieb E, Schultz T and Mattis D 1961 Ann. Phys. 16407

[19] Katsura S 1962 Phys. Rev. 1271508

[20] McCoy B M 1968 Phys. Rev. 173531

[21] Nonomura Y 1998 J. Phys. Soc. Japan 675

[22] Okabe Y and Kikuchi M 1986 Phys. Rev. B 347896

[23] Nonomura Y to be submitted to Phys. Rev. Lett.

[24] Suzuki M 1976 Phys. Lett. A 58435

[25] Suzuki M 1977 Prog. Theor. Phys. 831142

[26] Stauffer D 1992 Physica A 186197

[27] Ito N 1993 Physica A 192604

[28] Ito N 1993 Physica A 196591

[29] Sandvik A W and Vekić M 1995 Phys. Rev. Lett. 741226

[30] Nonomura $\mathrm{Y}$ in preparation

\section{Figure captions}

Figure 1: The checkerboard decomposition in one-dimensional systems.

Figure 2: Schematic behavior of the non-equilibrium relaxation of the order parameter from a perfectly-ordered state. Values of the order parameter are plotted versus Monte Carlo steps in a logarithmic scale (a) in the ordered phase, (b) at the transition point, and (c) in the disordered phase.

Figure 3: The order parameter of the present model is plotted versus Monte Carlo steps in a logarithmic scale (a) for $L=4096$ at $g=0.996 \sim 1.004$ and (b) for $L=1024,2048$ and 4096 at $g=1.000$. Solid lines are drawn as visual guides in the first figure.

Figure 4: The order parameter of the present model is plotted versus Monte Carlo steps in a logarithmic scale for (a) $L=32$ and (b) $L=64$. The crossover from 
"quasi-infinite" properties to finite-size properties is clearly seen. The crossover time scale is shown by the dashed line in each figure.

Figure 5: The order parameter of the present model is plotted versus Monte Carlo steps in a logarithmic scale (a) for $L=4096$ and $M=1024$ at $g=0.996 \sim 1.004$ and (b) for $L=2048$ and $M=2048$ at $g=0.996 \sim 1.004$. Solid lines are drawn as visual guides.

Figure 6: Estimates of the exponent $\lambda$ for various ranges of time scales $\left(t=t_{\text {ini }} \sim t_{\text {fin }}\right.$, $\Delta t=t_{\text {fin }}-t_{\text {ini }}=50,100$ and 200) are plotted versus $1 / t_{\text {mid }}$, with $t_{\text {mid }}=\left(t_{\text {ini }}+t_{\text {fin }}\right) / 2$. The solid, dotted and bold-solid curves correspond to the results for $\Delta t=50,100$ and 200, respectively. The final estimate $\lambda_{\text {est }}=0.1167 \pm 0.0033$ is displayed by straight lines.

Figure 7: Ground-state averages of (a) the squared magnetization and (b) the susceptibility at the transition point are plotted versus system sizes in a logarithmic scale. The solid lines are drawn by the least-squares fitting of the data for $64 \leq L \leq 128$ with (11), and the dashed lines are obtained by the least-squares fitting of the data for $24 \leq L \leq 128$ with (16) and (17).

Figure 8: The susceptibility in the $L=128$ cluster is plotted versus the control parameter $g$ in the vicinity of the transition point. The slope of these data (the solid line) is evaluated by the least-squares fitting of the data drawn with full symbols.

Figure 9: The slopes of (a) the squared magnetization and (b) the susceptibility are plotted versus system sizes in a logarithmic scale. The solid lines are drawn by the least-squares fitting of the data for $80 \leq L \leq 128$ with (22).

Figure 10: The order parameter of the present model including the Trotter-direction global flip is plotted versus Monte Carlo steps in a logarithmic scale for $L=4096$. Solid lines are drawn as visual guides.

Figure 11: Size dependence of (a) the squared magnetization and (b) the susceptibility at the transition point in a logarithmic scale. The Trotter-direction global flip is included in these simulations. The solid lines are drawn by the least-squares fitting of the data for $64 \leq L \leq 128$ with (11), and the dashed lines are obtained by the least-squares fitting of the data for $32 \leq L \leq 128$ with (16) and (17). 


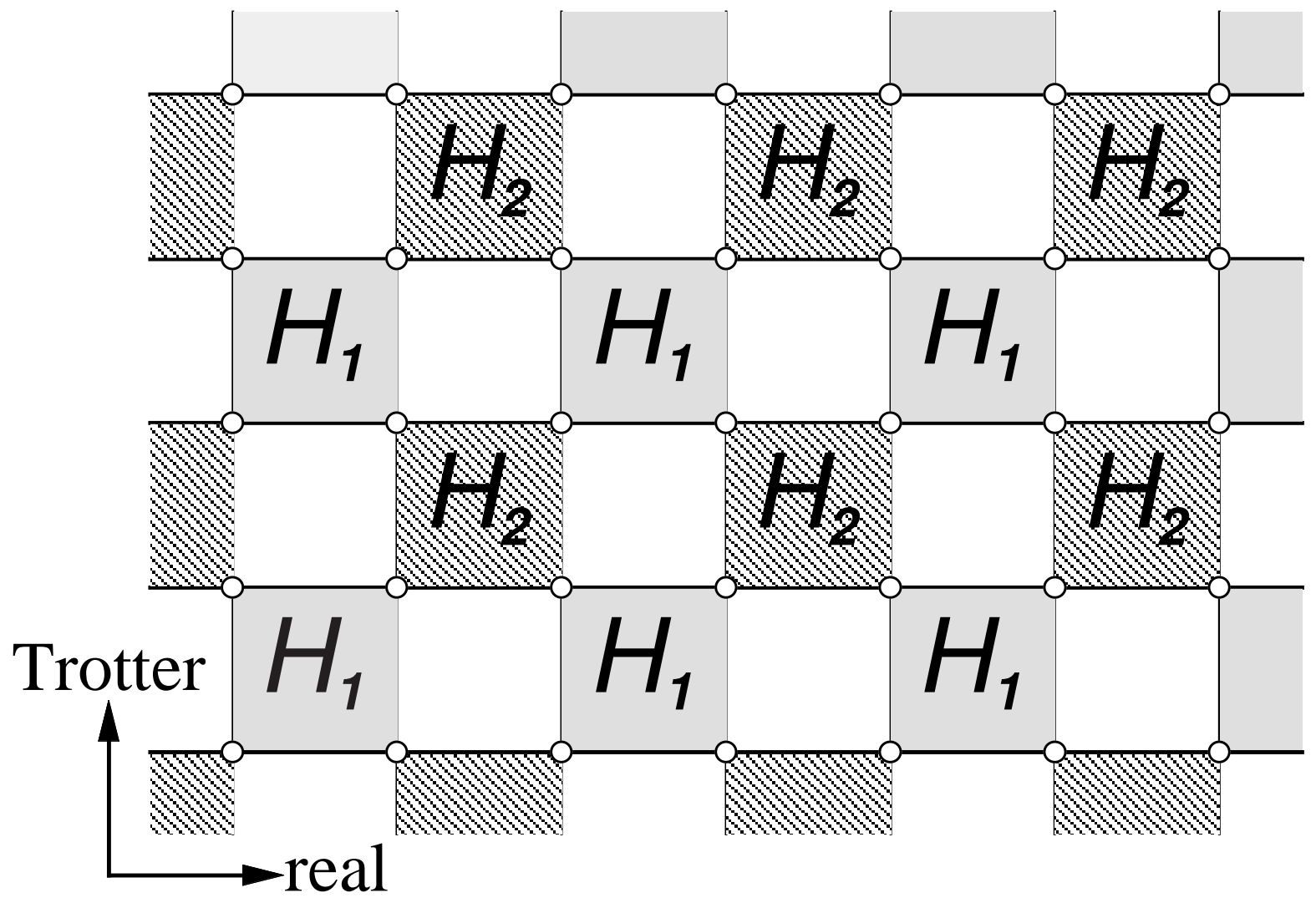

Fig.1: Y.Nonomura 
Fig.2: Y.Nonomura

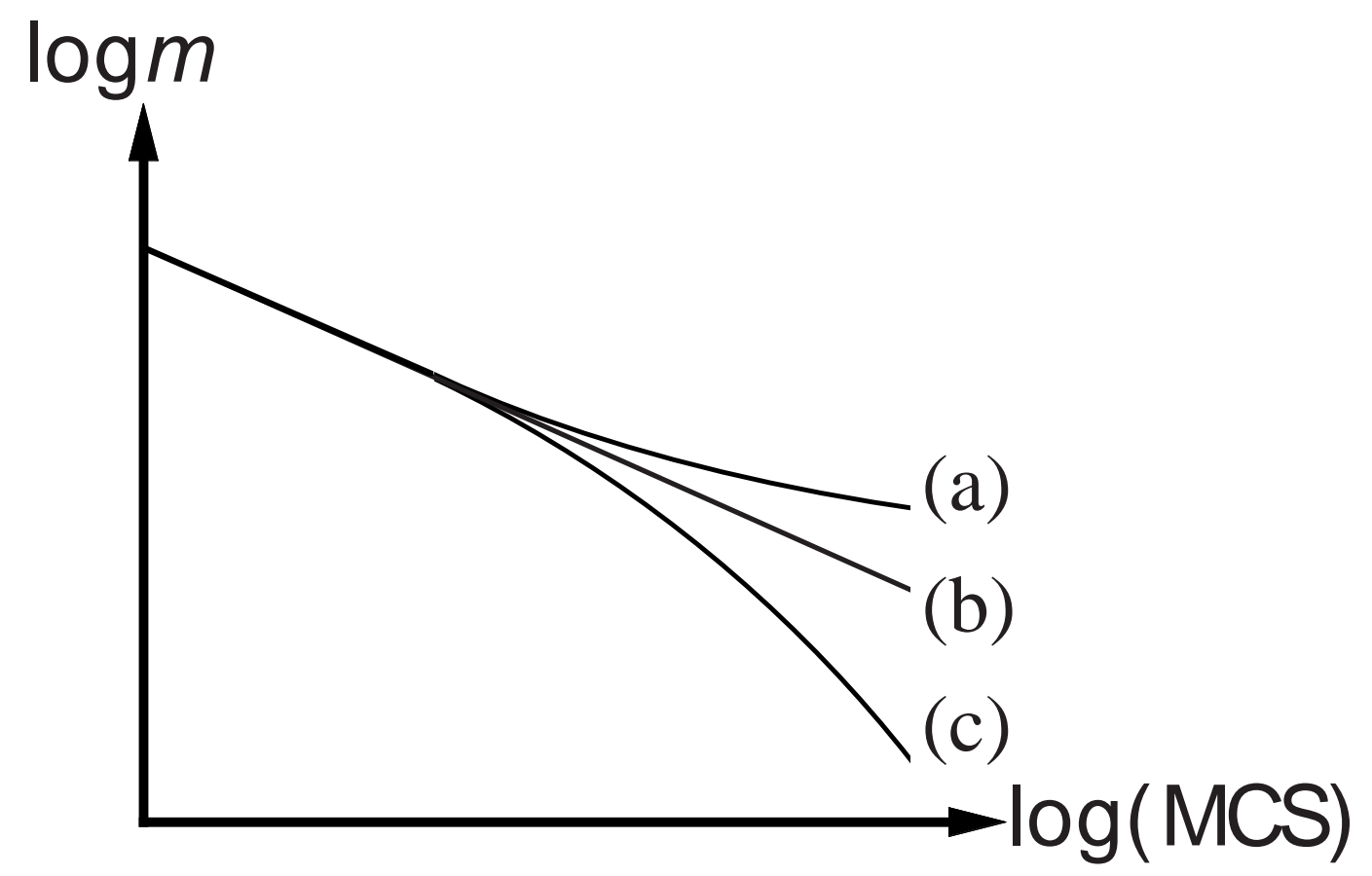


Fig.3(a): Y.Nonomura

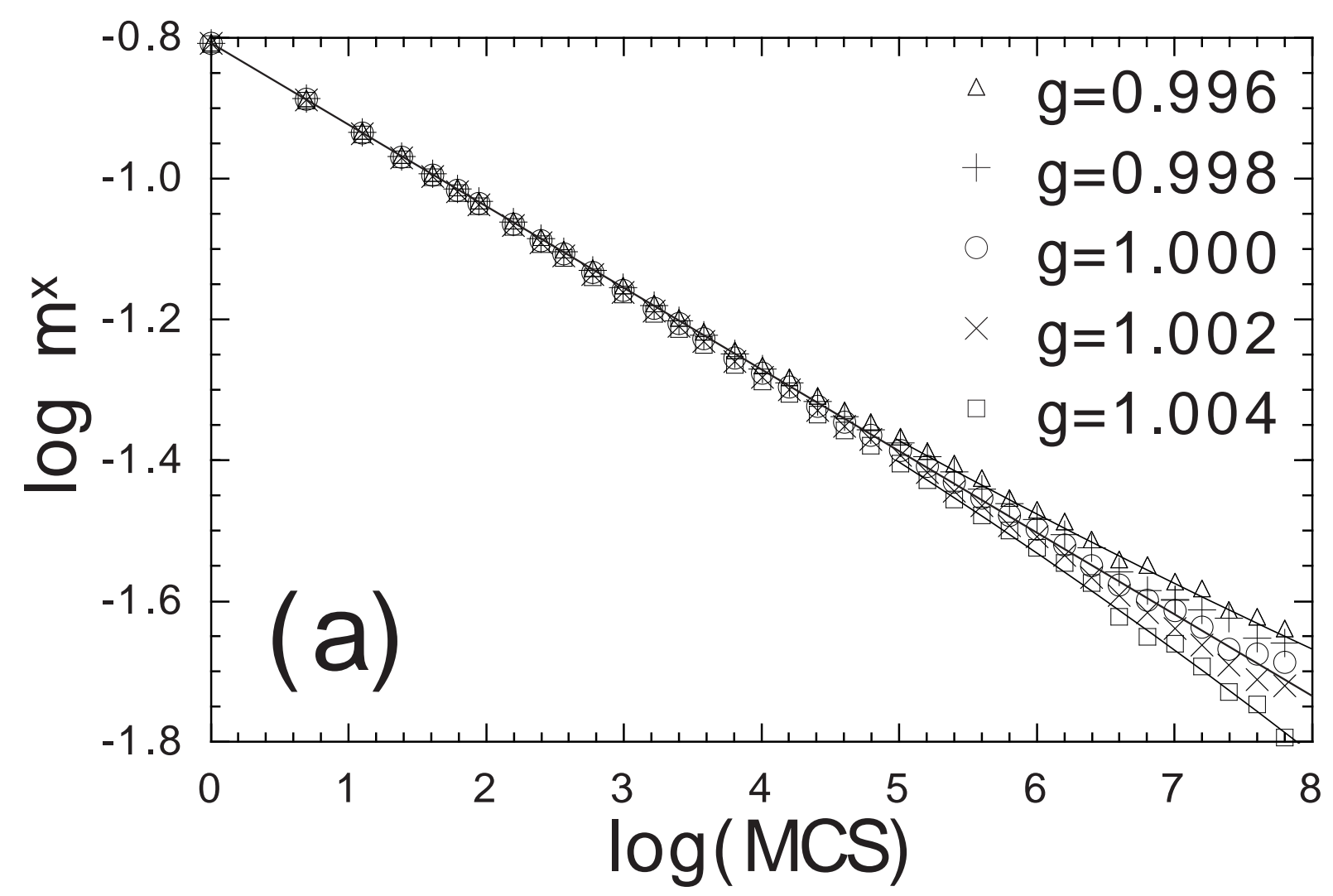


Fig.3(b): Y.Nonomura

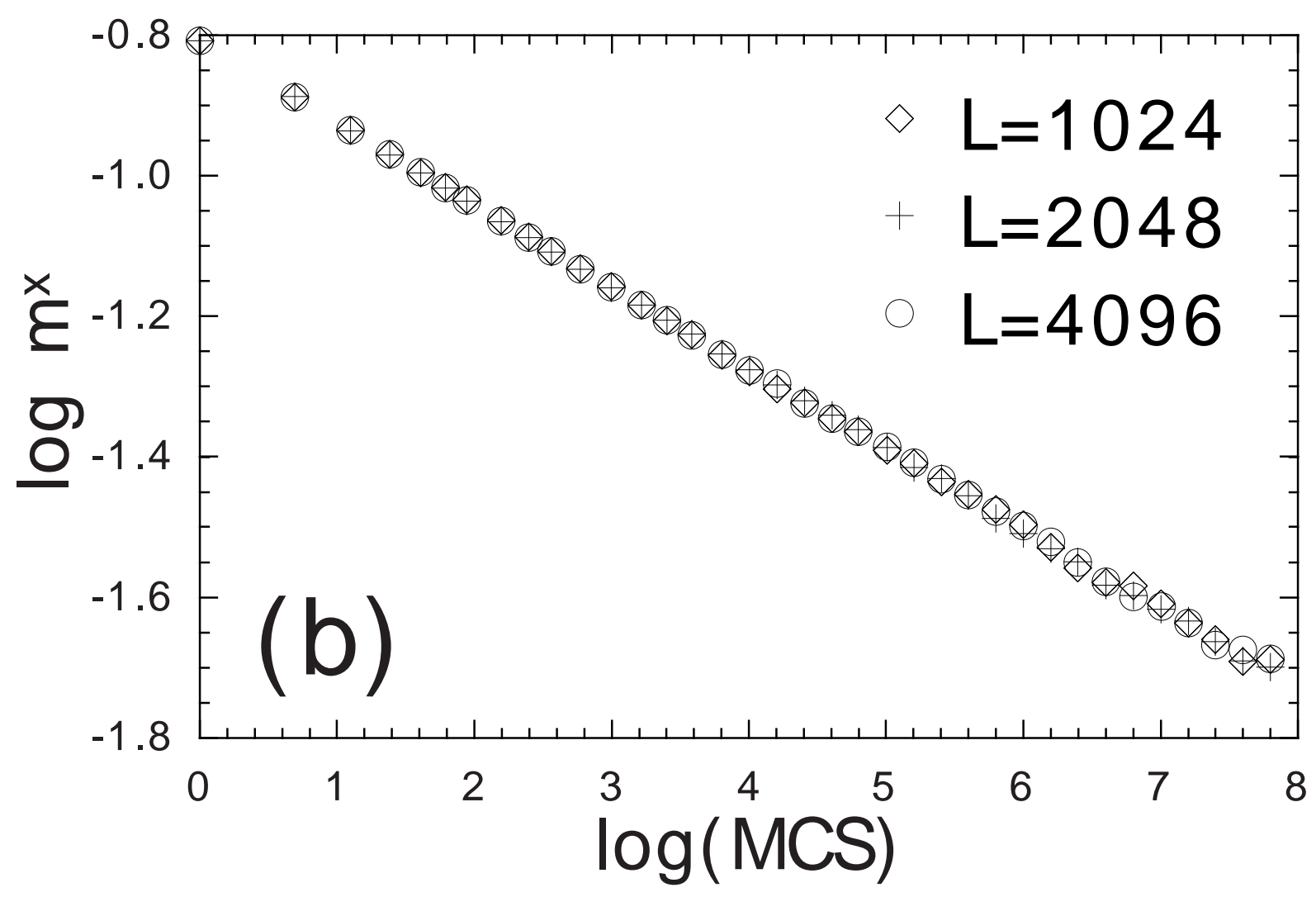


Fig.4(a): Y.Nonomura

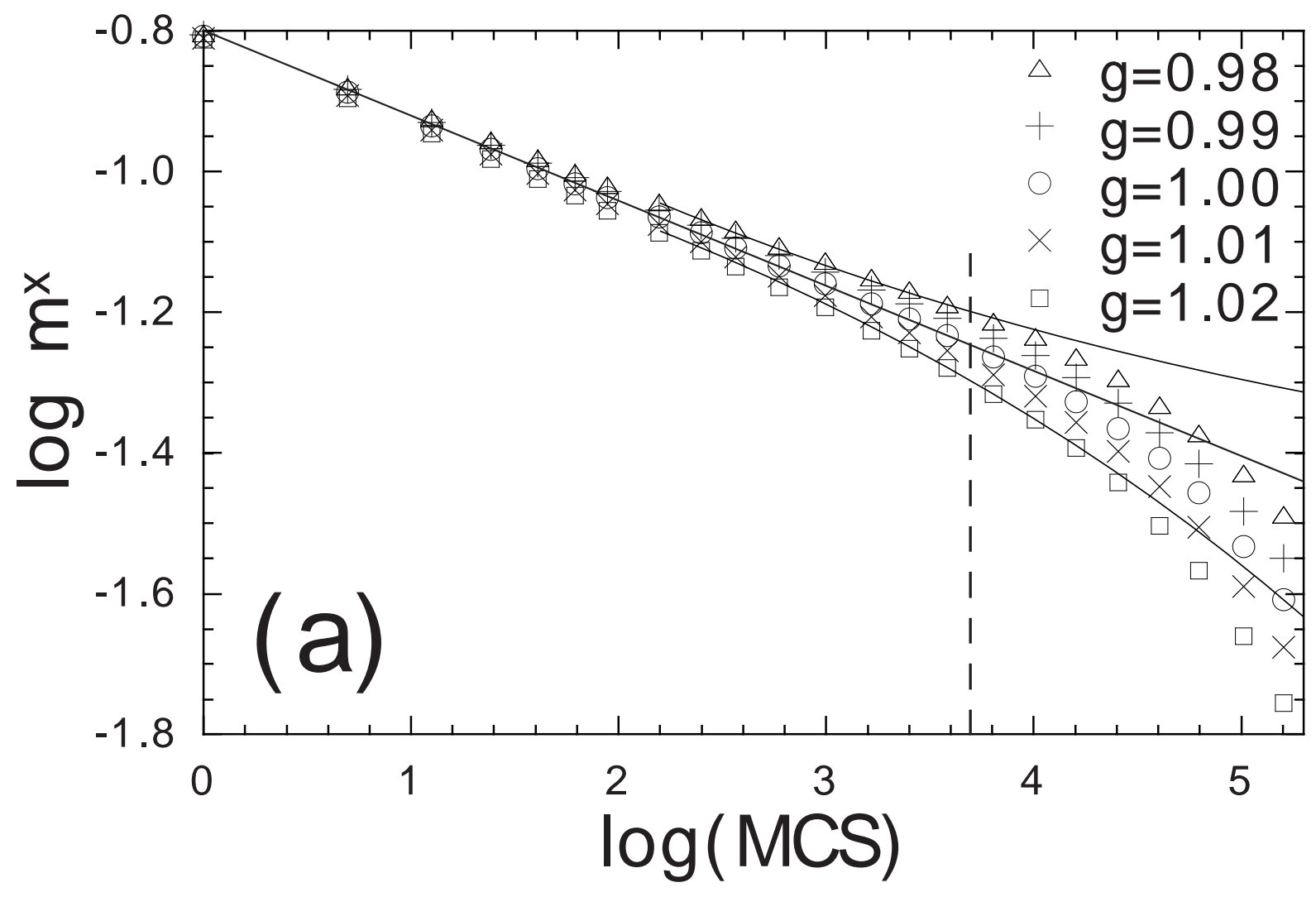


Fig.4(b): Y.Nonomura

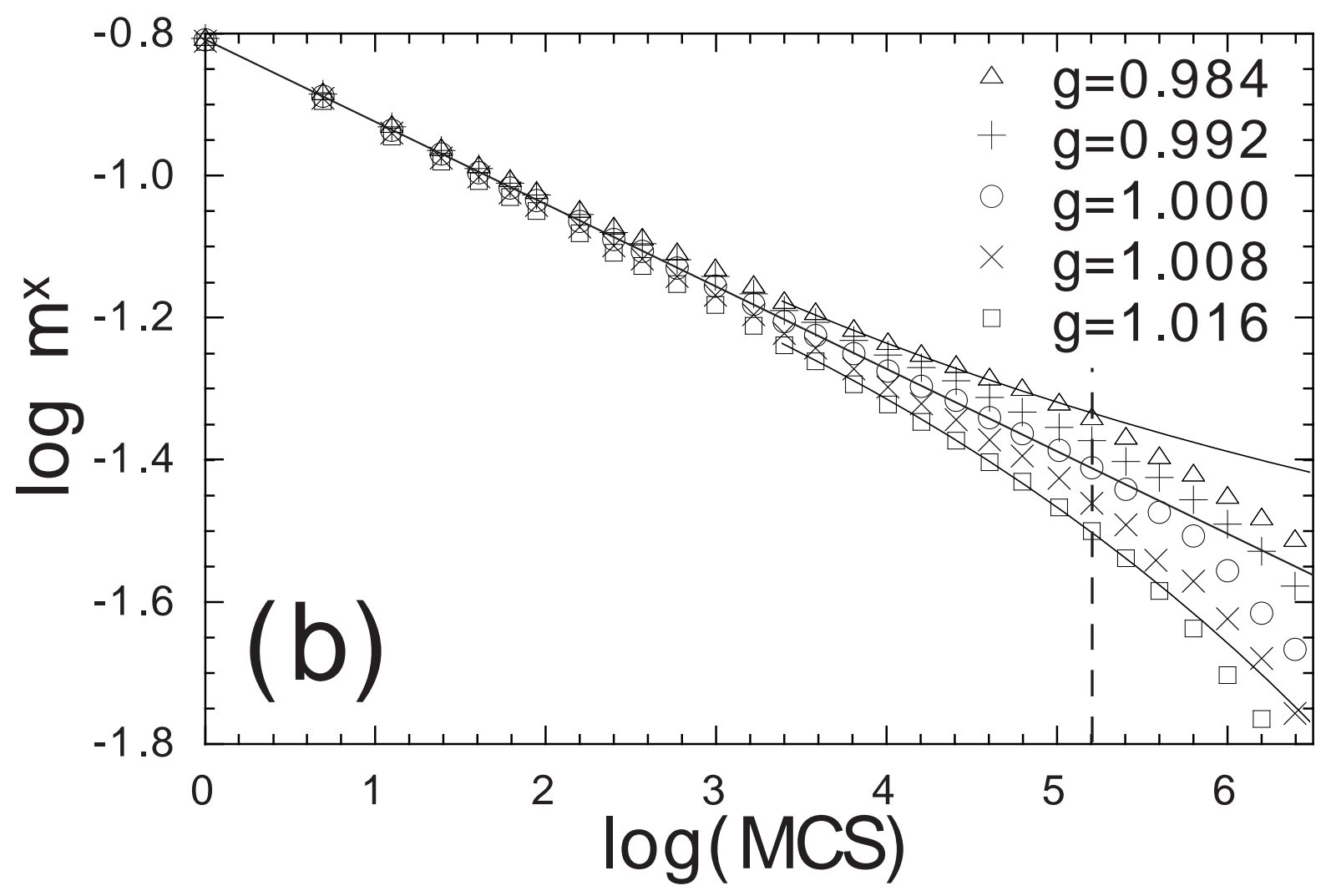


Fig.5(a): Y.Nonomura

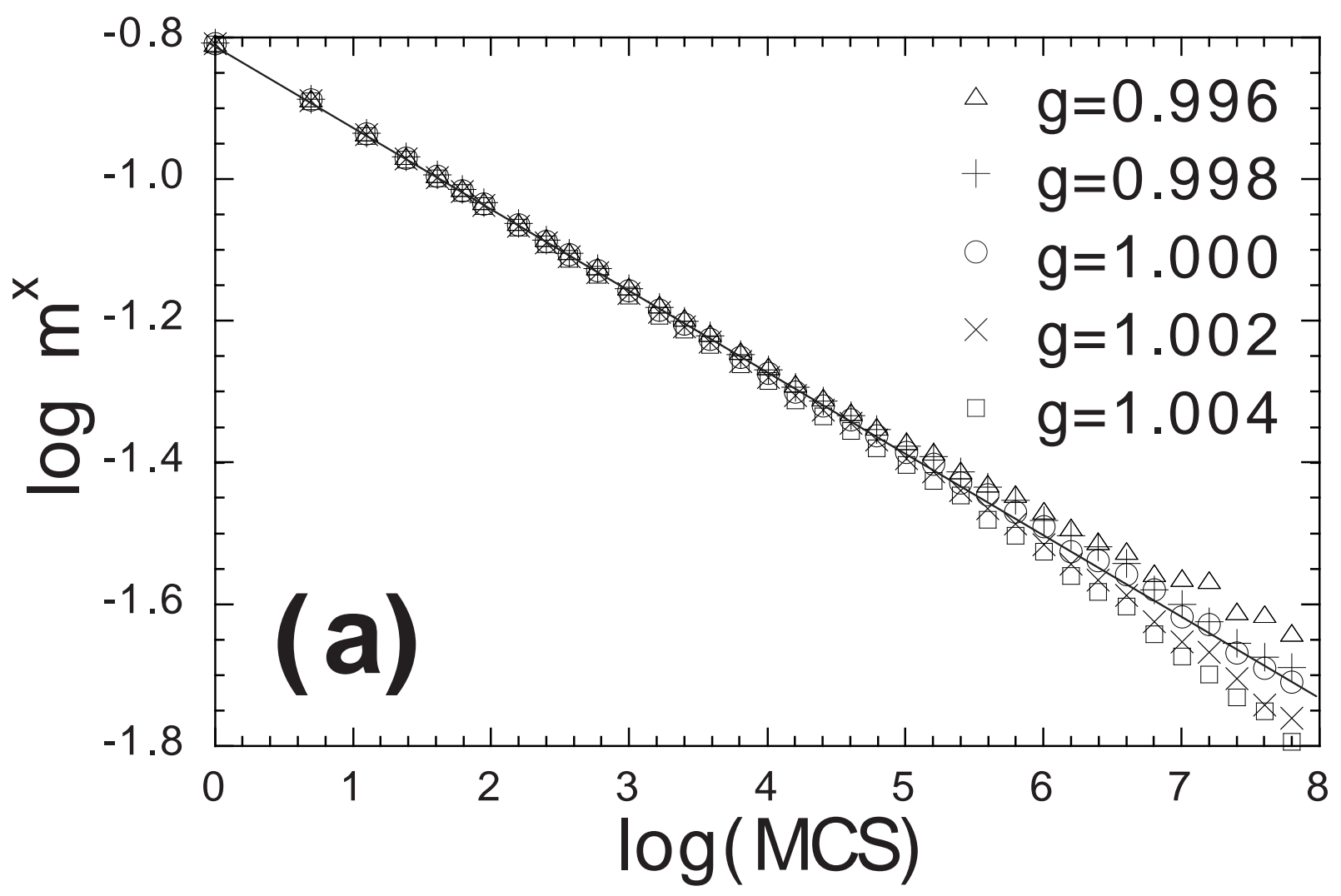


Fig.5(b): Y.Nonomura

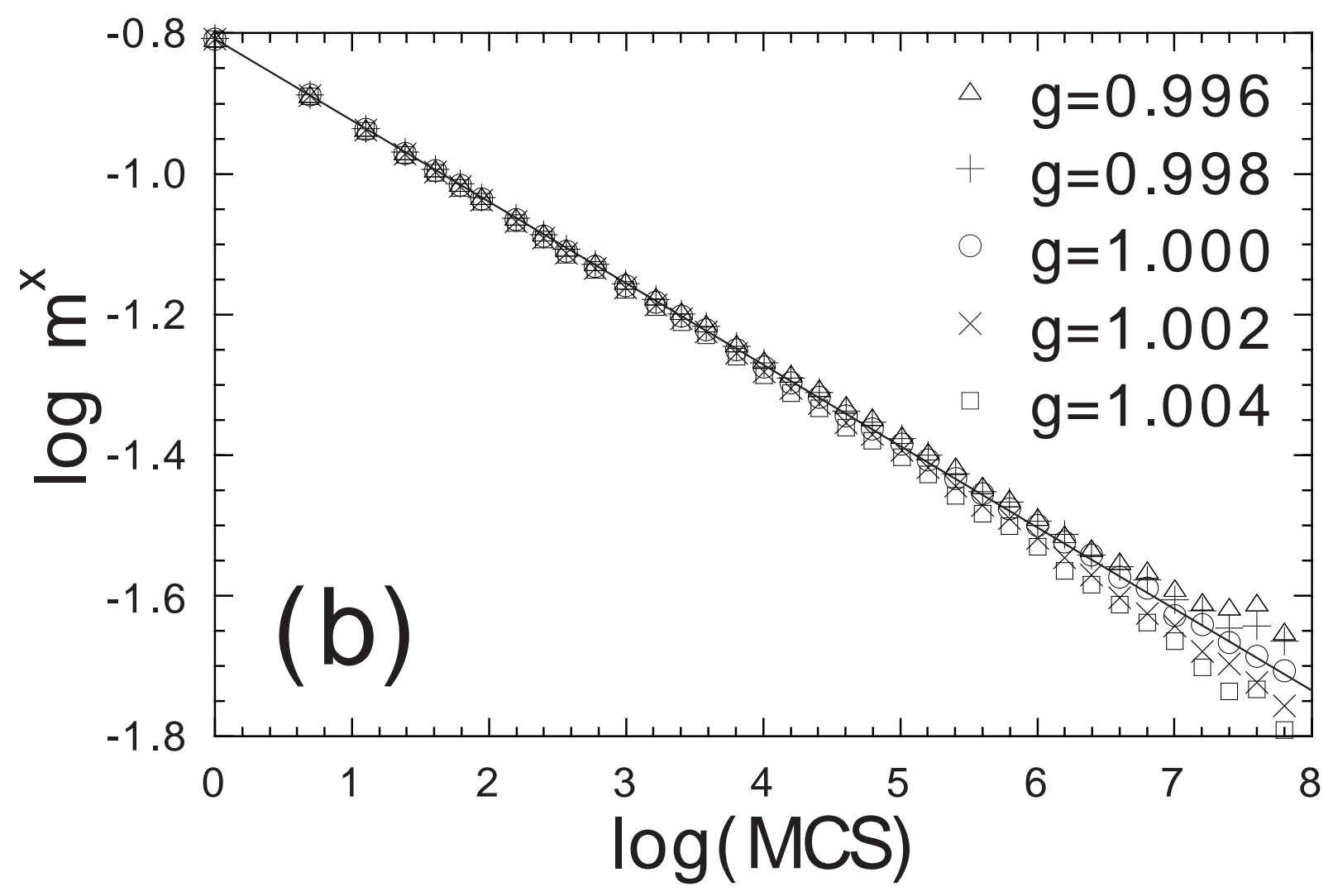


Fig. 6: Y.Nonomura

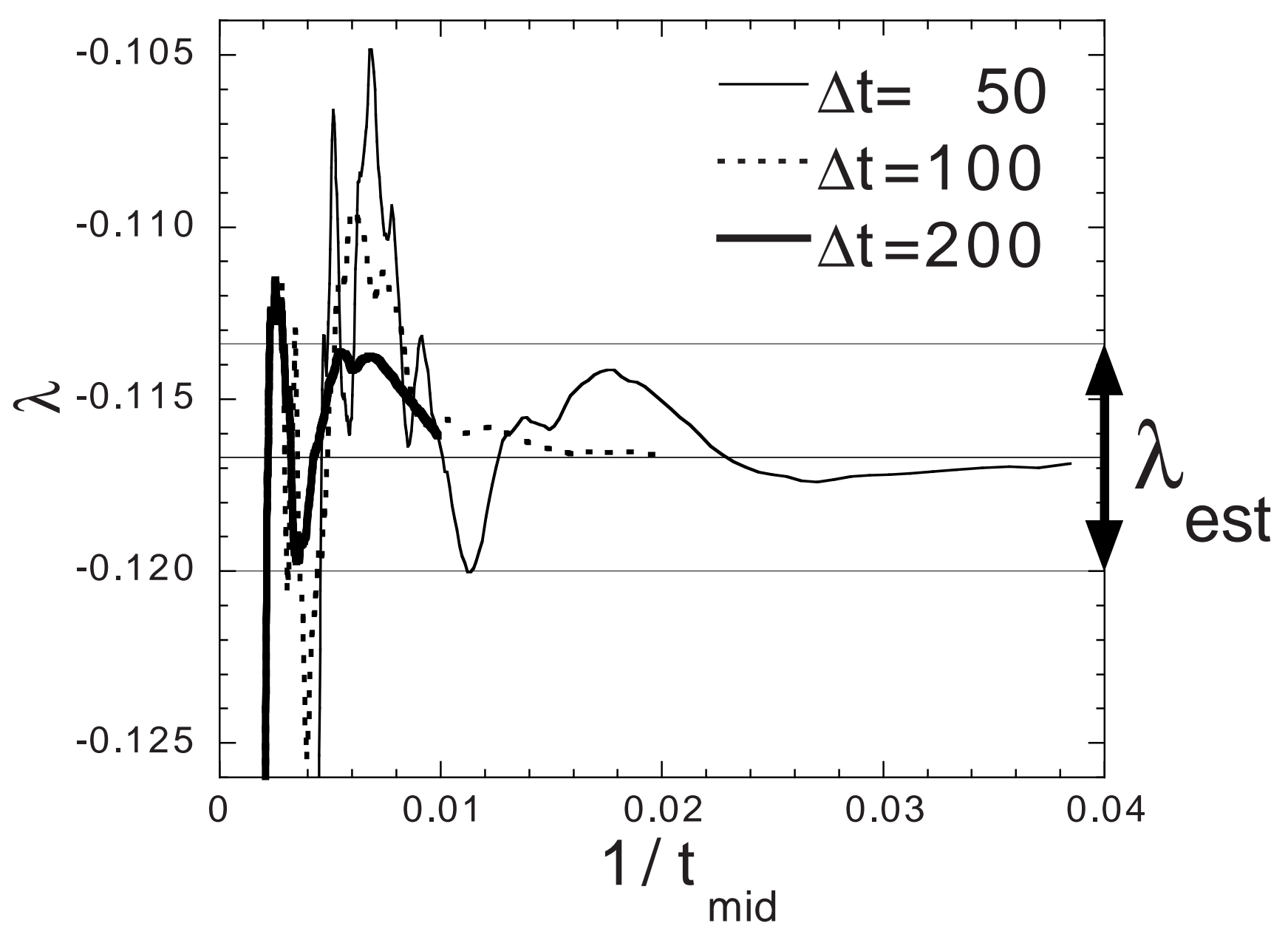


Fig.7(a): Y.Nonomura

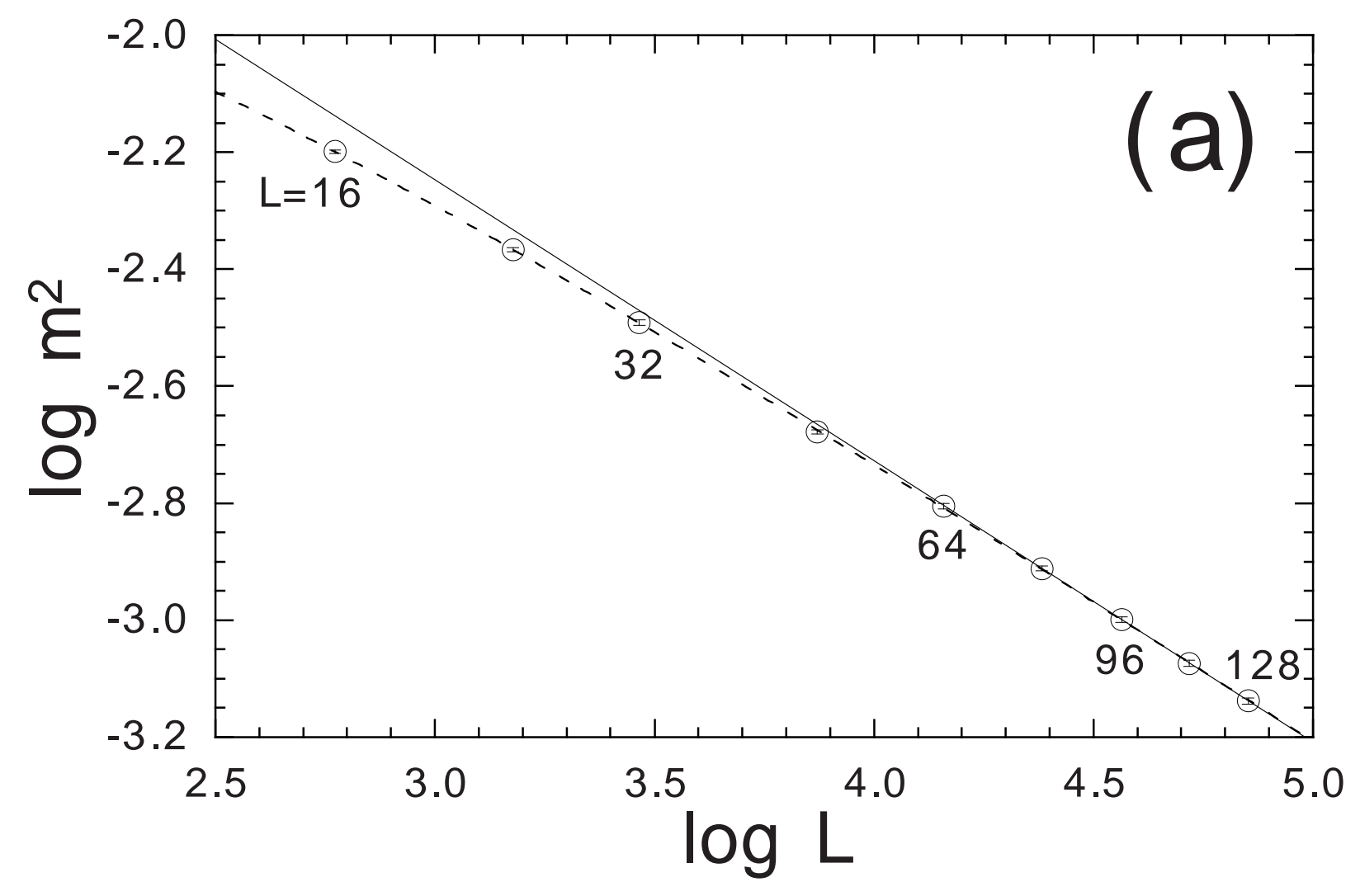


Fig.7(b): Y.Nonomura

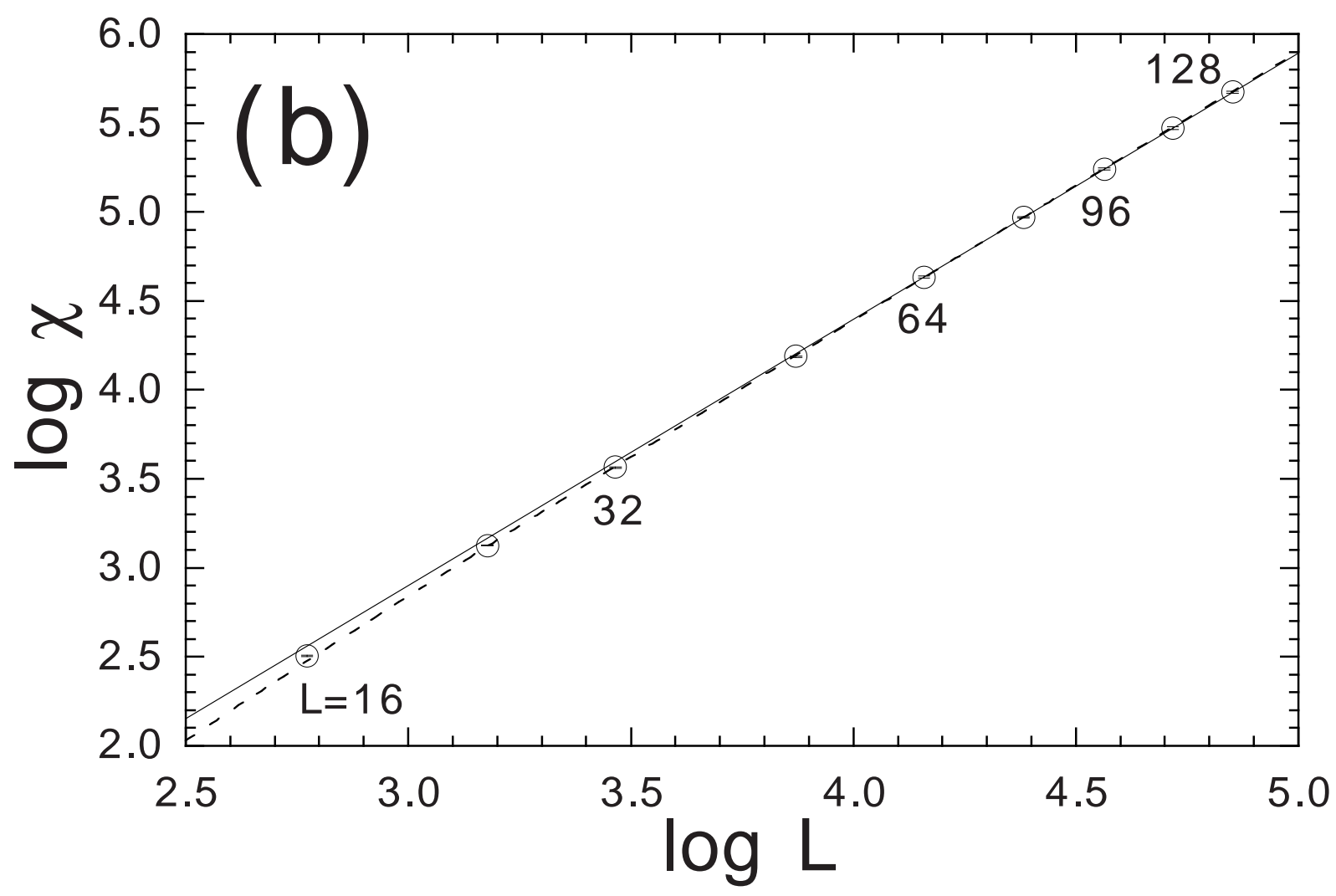


Fig.8: Y.Nonomura

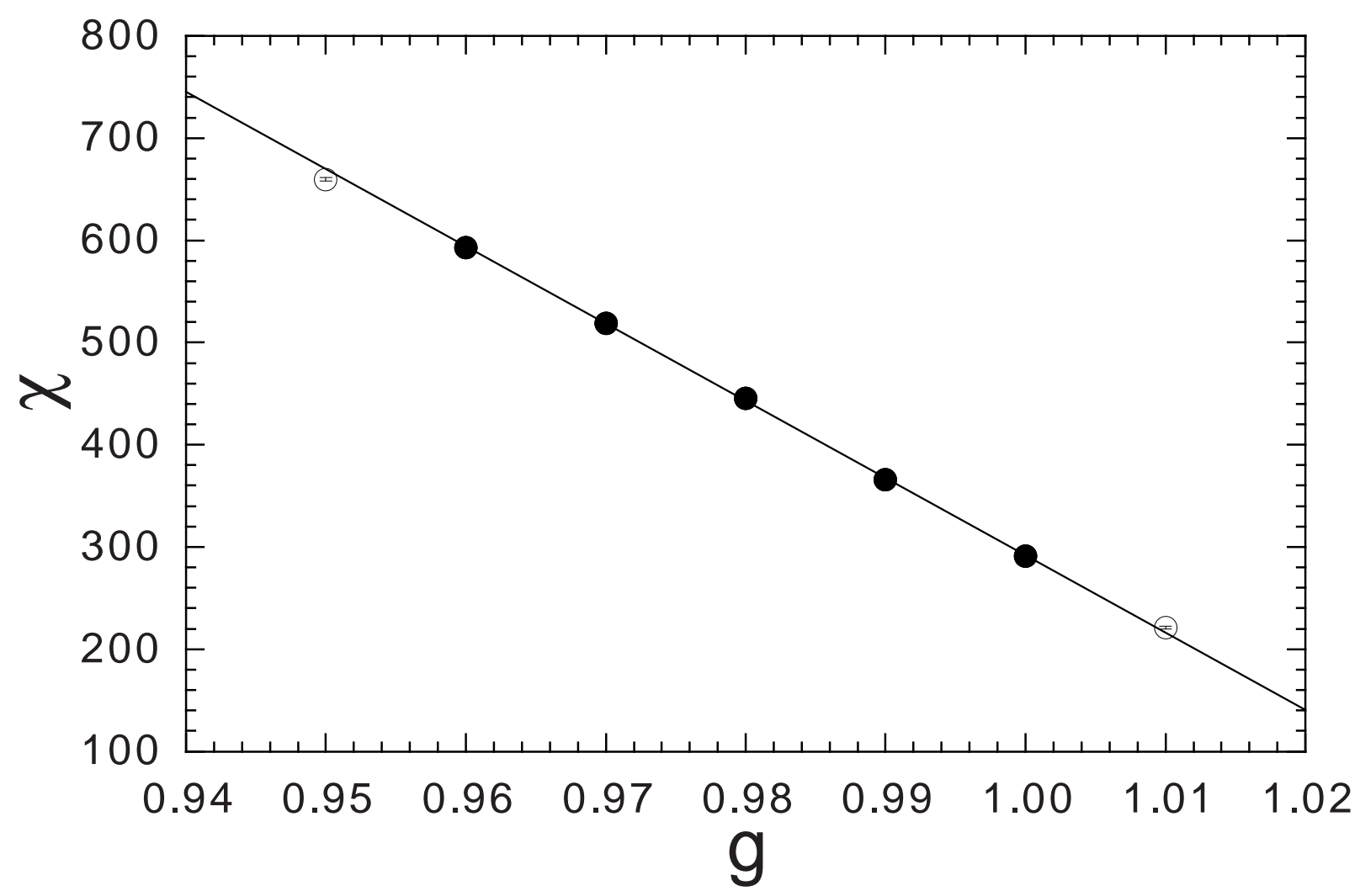


Fig.9(a): Y.Nonomura

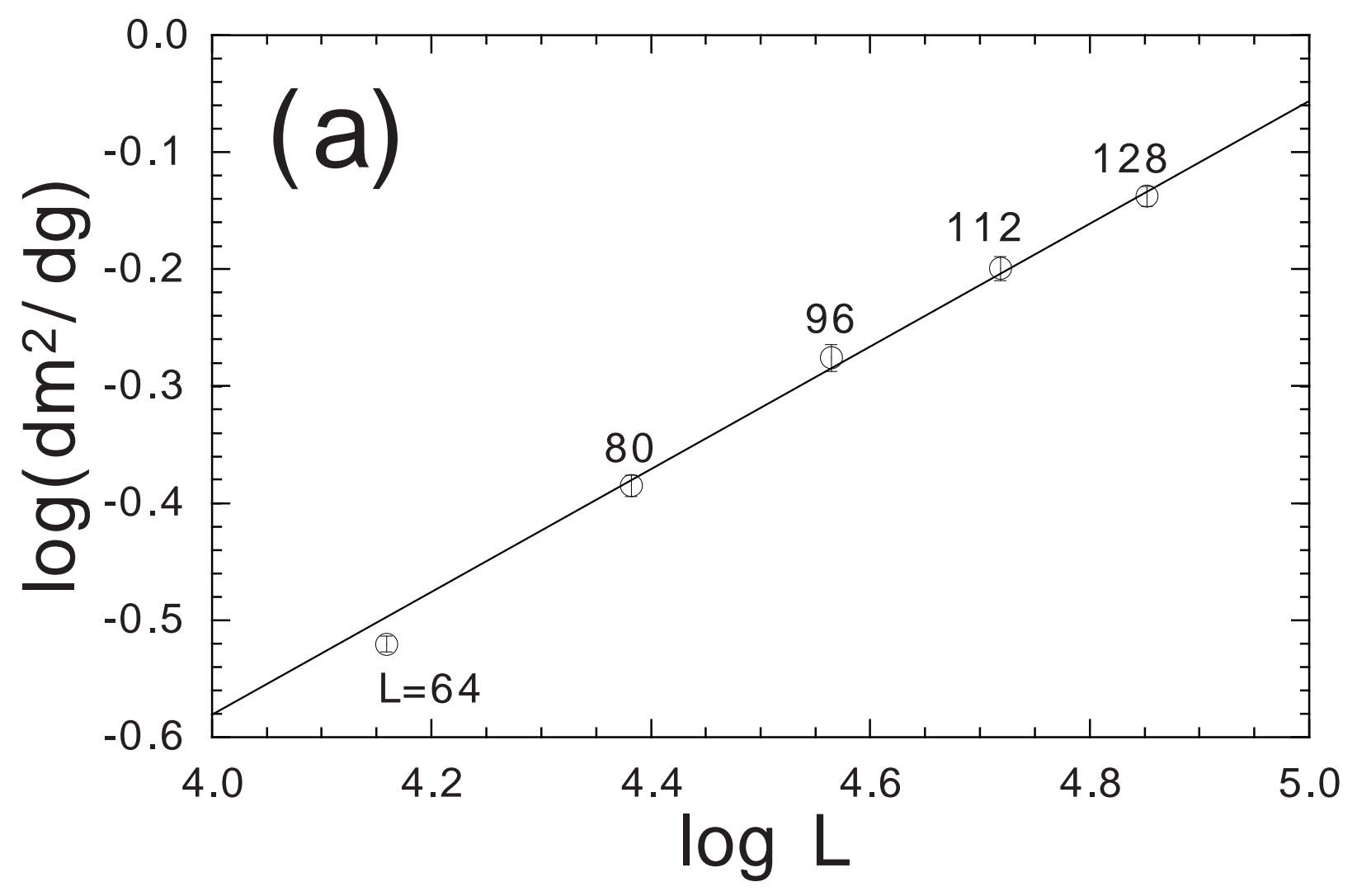


Fig.9(b): Y.Nonomura

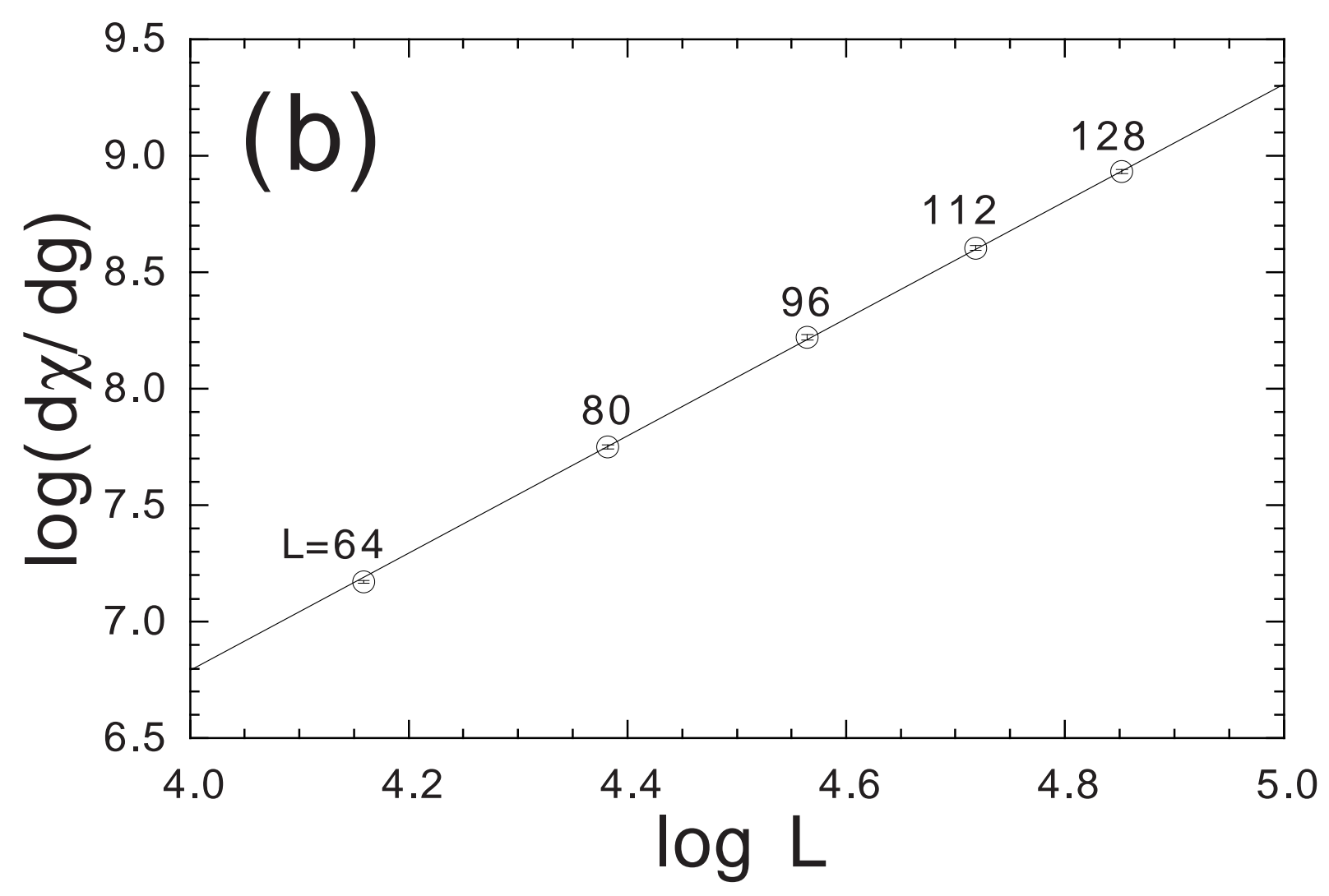


Fig.10: Y.Nonomura

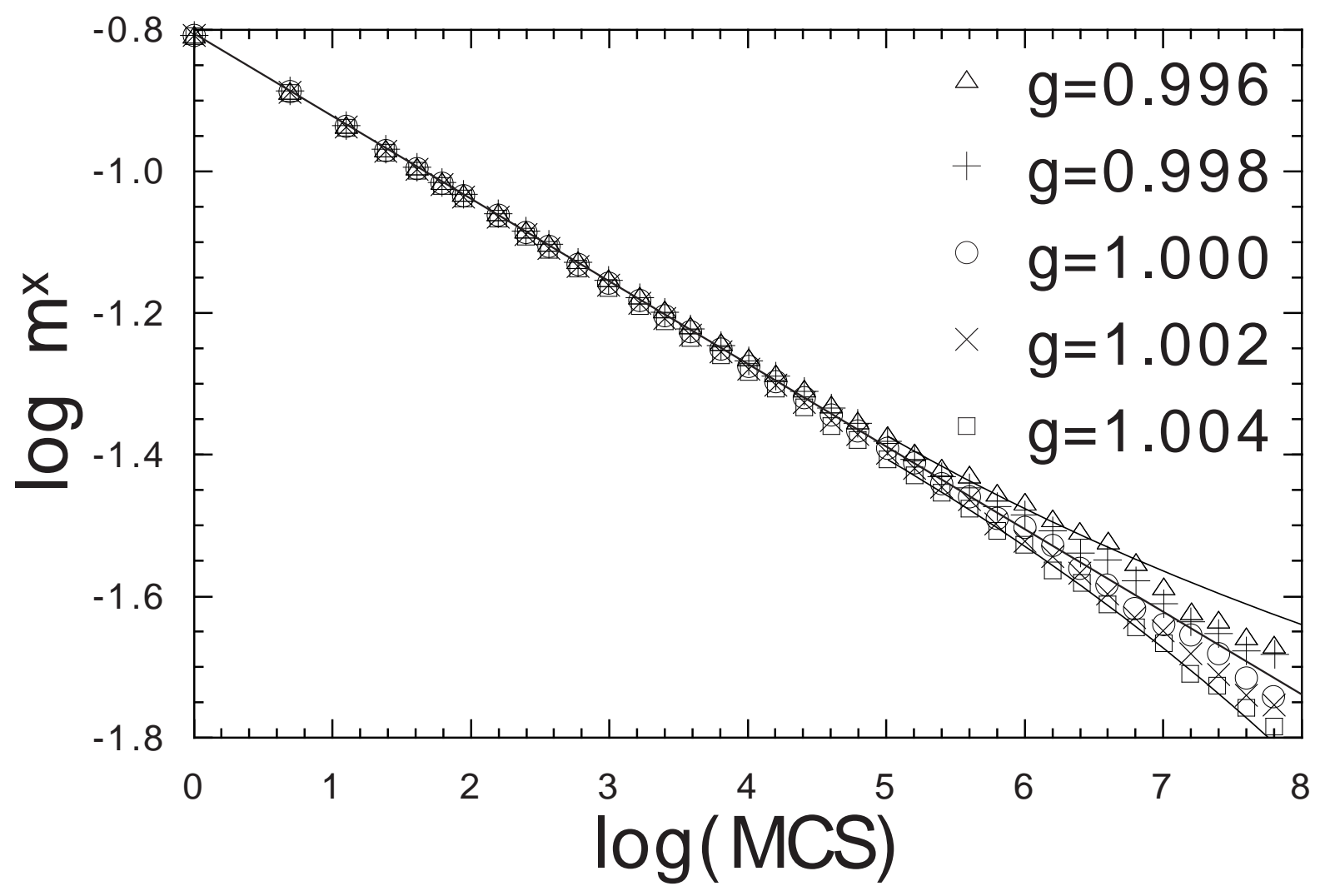


Fig.11(a): Y.Nonomura

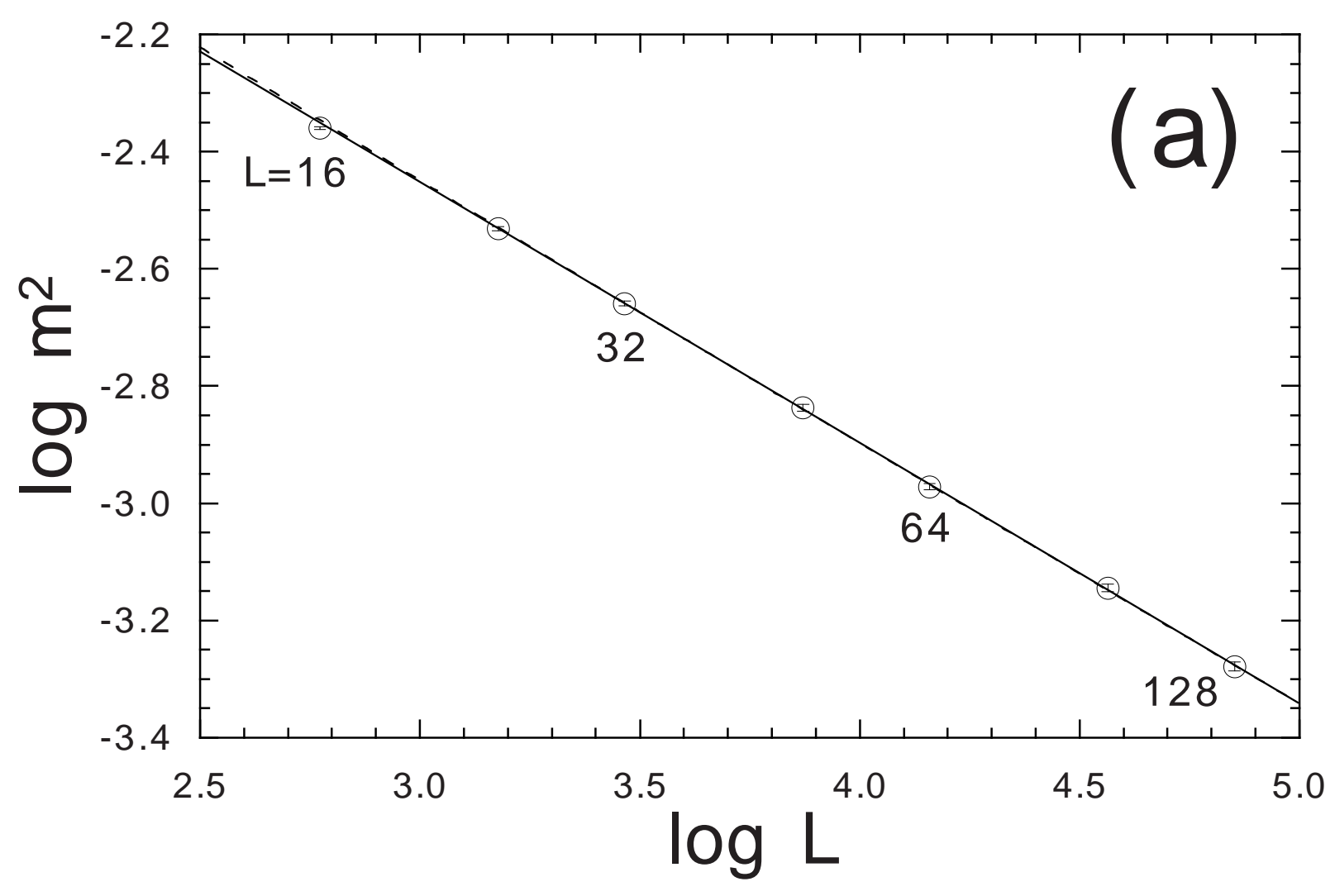


Fig.11(b): Y.Nonomura

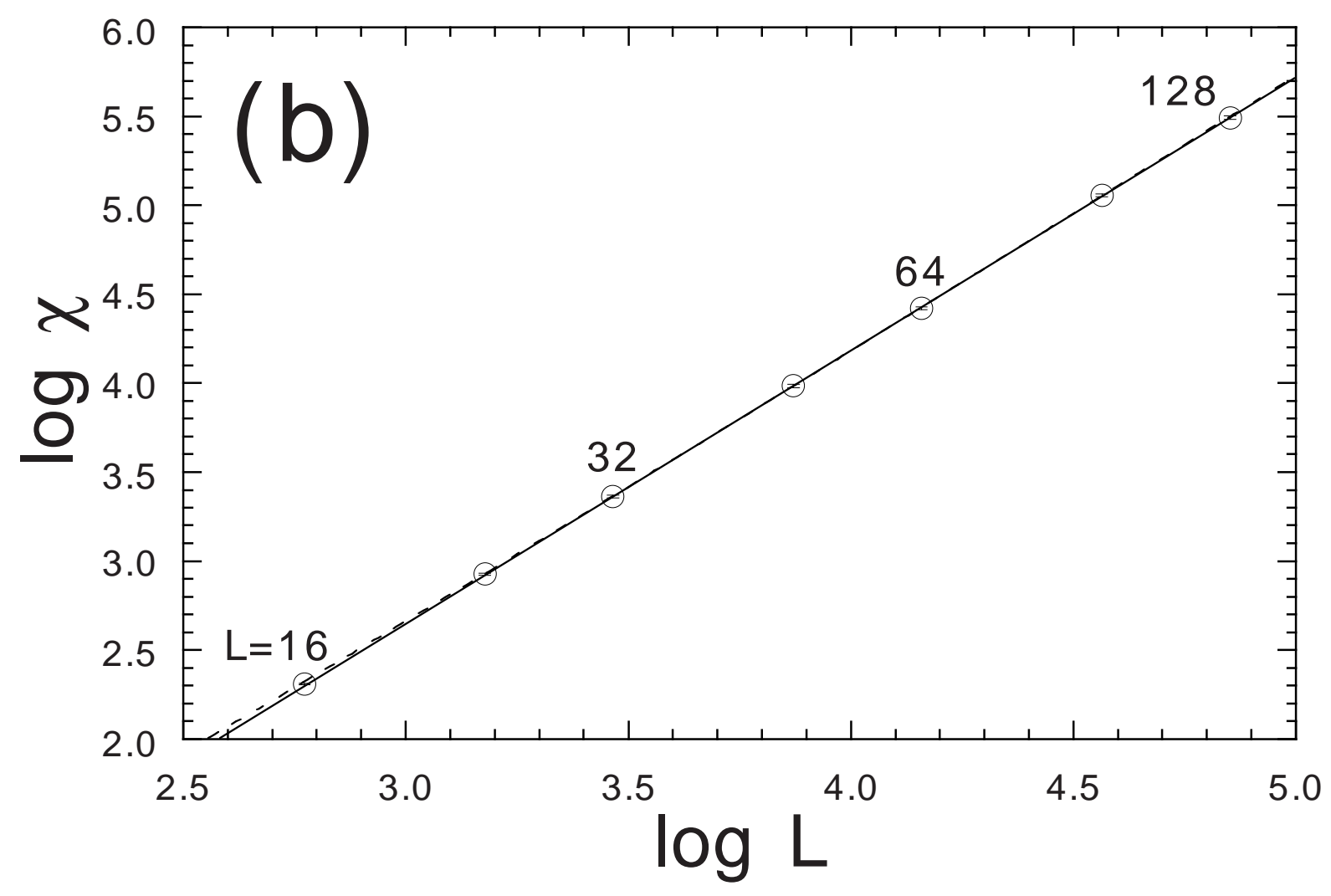

